\title{
Simulation of Finite-Source Retrial Queueing Systems with Collisions and Non-reliable Server
}

\author{
Ádám Tóth ${ }^{1(\varpi)}$, Tamás Bérczes ${ }^{1}$, János Sztrik $^{1}$, and Anna Kvach ${ }^{2}$ \\ 1 Faculty of Informatics, University of Debrecen, \\ Egyetem tér 1, Debrecen 4032, Hungary \\ adamtoth102@gmail.com, \{berczes.tamas, sztrik.janos\}@inf.unideb.hu \\ 2 Tomsk State University, Tomsk, Russia \\ kvach_as@mail.ru
}

\begin{abstract}
The aim of the present paper is to build a simulation program to investigate finite-source retrial queuing system with collision of the customers where the server is subject to random breakdowns and repairs depending on whether it is idle or busy. All the random variables involved in the model construction are assumed to be independent and generally distributed. The novelty of the investigation is to carry sensitivity analysis of the performance measures using various distributions. Several figures show the effect of different distributions on the performance measures such as mean and variance of number of customers in the system, mean and variance of response time, mean and variance of time a customer spent in service, mean and variance of sojourn time in the orbit.
\end{abstract}

Keywords: Simulation - Sensitivity analysis · Finite-source queuing system $\cdot$ Closed queuing system $\cdot$ Collision $\cdot$ Unreliable server $\cdot$ Retrial queue

\section{Introduction}

Retrial queues have been commonly used to depict many real situations emerging in telephone switching systems, telecommunication networks, computer networks and computer systems, call centers, wireless communication systems, etc. In many practical situations it is important to bear in mind that the rate of generation of new calls decreases as the number of customers in the system increases. This can be achieved with the use of finite-source, or quasi-random input models. Retrial queues with quasi-random input are recent interest in modeling cellular mobile networks, computer networks and local-area networks with random access protocols, and with multiple-access protocols, see, for example, [3,8].

In practice a few components of the system are prone to random breakdowns so it is important to study reliability of retrial queues with server breakdowns and repairs. Due to this it has a heavy influence on the performance measures of the system. Finite-source retrial queues with unreliable server have been investigated in several recent papers for example, [2, 5, 7, 13,14].

(C) Springer International Publishing AG 2017

V.M. Vishnevskiy et al. (Eds.): DCCN 2017, CCIS 700, pp. 146-158, 2017.

DOI: $10.1007 / 978-3-319-66836-9 \_13$ 
In many cases including data transmission from disparate sources there is a possibility to be conflict for a limited number of channels or other facilities. Several sources launching uncoordinated attempts can produce collisions leading to the loss of the transmission and consequently the necessity for retransmission. An essential matter is to develop workable procedures for allaying the conflict and corresponding message delay. There have been recent results on retrial queues with collision in $[1,4,9-12]$.

The aim of the present paper is to investigate such systems with unreliable server which are finite source, and collisions can take place. In this paper we build simulation models using SimPack, a collection of $\mathrm{C} / \mathrm{C}++$ libraries and executable programs for computer simulation [6], to receive the desired performance measures. In this collection various algorithms are supported connected with simulation including discrete event simulation, continuous simulation and combined (multi-model) simulation. The novelty of this work is to provide sensitivity analysis using various distributions.

\section{System Model}

Let us consider a finite source retrial queueing system in which the number of sources is denoted by $N$ and each of them can generate request with rate $\lambda / N$, that is the source time is exponentially distributed with parameter $\lambda / N$. If a customer finds the server idle it enters into service instantly. The service times are supposed to be gamma distributed with parameter $\alpha$ and $\beta$. When the server is engaged with a request, an arriving (from the orbit or the source) customer evokes a collision with a customer under service and both requests are directed towards the orbit. From the orbit it retries to be served after an exponentially distributed time with parameter $\sigma / N$. It is supposed that the service unit fails after some time which is an exponentially distributed random variable with parameter $\gamma_{0}$ when it is busy and with parameter $\gamma_{1}$ when it is idle.

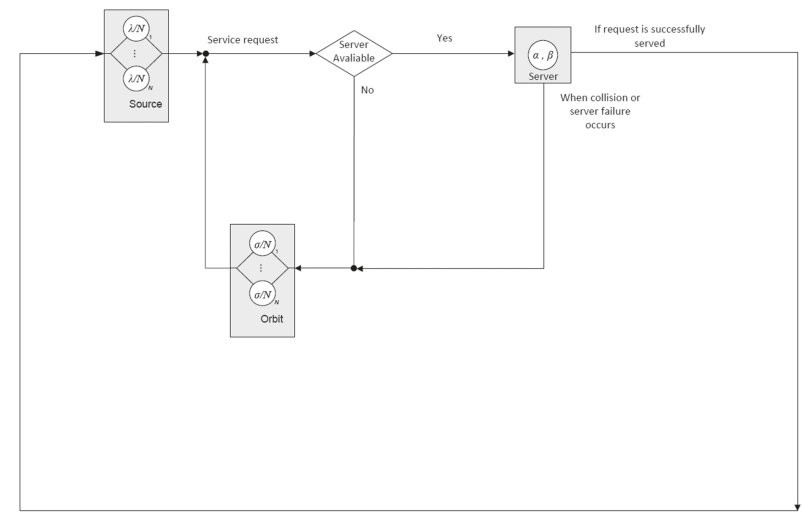

Fig. 1. System model 
Immediately upon the breakdown it is forwarded for repair and the restoration time is also exponentially distributed random variable with parameter $\gamma_{2}$. We suppose that when the server is unavailable every source is eligible to generate customers and sends it to the unit, and the customers from the orbit may retry to the server. Moreover, in this model we suppose that the interrupted request gets into the orbit instantaneously and all of its services are independent of each other. When the submission is successful, the requests go back to the source. All the random variables involved in the model construction are assumed to be independent of each other (Fig. 1).

\section{Simulation Results}

\subsection{Scenario A}

The following table shows the input parameters of Scenario A (see Table 1).

Table 1. Numerical values of model parameters

\begin{tabular}{l|l|l|l|l|l|l|l|l}
\hline Case & $\mathrm{N}$ & $\lambda / N$ & $\gamma_{0}$ & $\gamma_{1}$ & $\gamma_{2}$ & $\sigma / N$ & $\alpha$ & $\beta$ \\
\hline 1 & 100 & 0.01 & 0.1 & 0.1 & 1 & 0.01 & 0.5 & 0.5 \\
\hline 2 & 100 & 0.01 & 0.1 & 0.1 & 1 & 0.01 & 1 & 1 \\
\hline 3 & 100 & 0.01 & 0.1 & 0.1 & 1 & 0.01 & 2 & 2 \\
\hline
\end{tabular}

Figure 2 shows the steady-state distribution of the three investigated cases. It is observed the mean number of customers increases as $\alpha$ and $\beta$ are getting larger. Case 2 is a special case because when $\alpha=1$ it represents the exponential distribution. From the shape of the curves it is clearly visible that the steadystate distribution of the cases are normally distributed. The next table presents the considered performance measures in relation with the different cases (see Table 2).

In Table 2 the notations mean the followings: $E(N S)$ and $D^{2}(N S)$ - mean number and variance of customers, $E(T)$ and $D^{2}(T)$ - mean and variance of response time, $E(W)$ and $D^{2}(W)$ - mean and variance of waiting time, $E(S)$ and $D^{2}(S)$ - mean and variance of successful service time, $E(I S)$ - mean interrupted service time.

Table 2. Numerical results

\begin{tabular}{l|l|l|l|r|l|r|r|r|l}
\hline Case & $\mathrm{E}(\mathrm{NS})$ & $D^{2}(N S)$ & $\mathrm{E}(\mathrm{T})$ & $D^{2}(T)$ & $\mathrm{E}(\mathrm{W})$ & $D^{2}(W)$ & $\mathrm{E}(\mathrm{S})$ & $D^{2}(S)$ & $\mathrm{E}(\mathrm{IS})$ \\
\hline 1 & 63.6842 & 27.9734 & 175.3073 & 65657.3454 & 174.5884 & 65434.6696 & 0.3147 & 0.1979 & 0.4041 \\
\hline 2 & 70.5912 & 24.3012 & 239.9734 & 105273.4267 & 238.9734 & 104918.6389 & 0.4784 & 0.2289 & 0.5217 \\
\hline 3 & 75.1825 & 21.2439 & 302.8106 & 151781.1411 & 301.5377 & 151277.6006 & 0.6472 & 0.2095 & 0.6257 \\
\hline
\end{tabular}




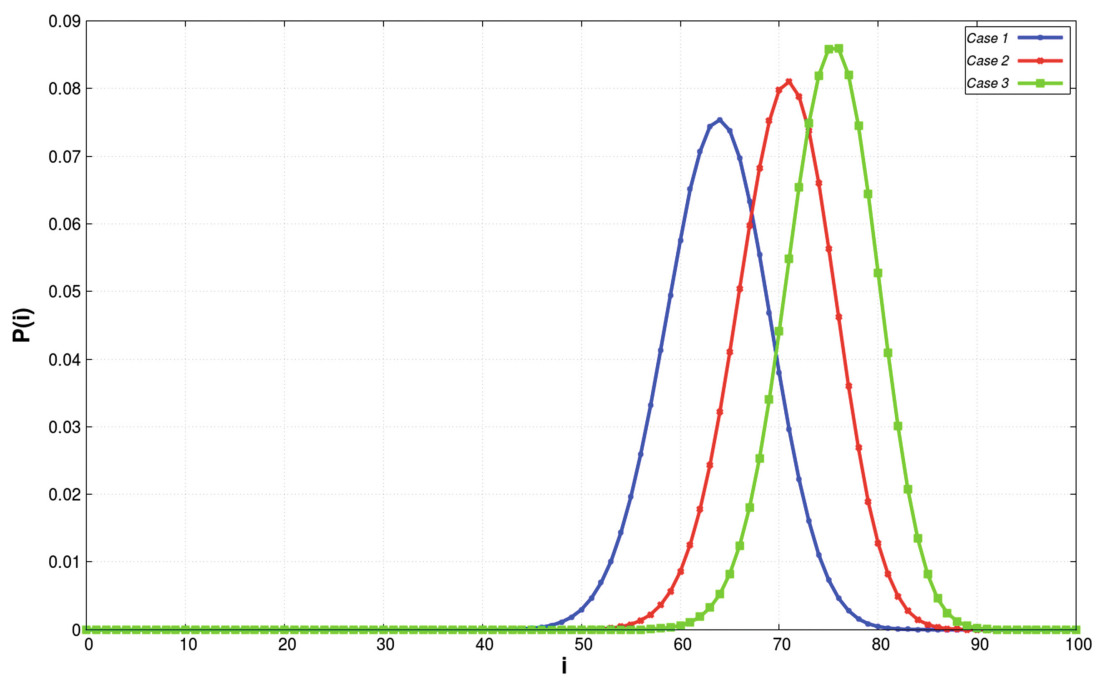

Fig. 2. Comparison of steady-state distributions

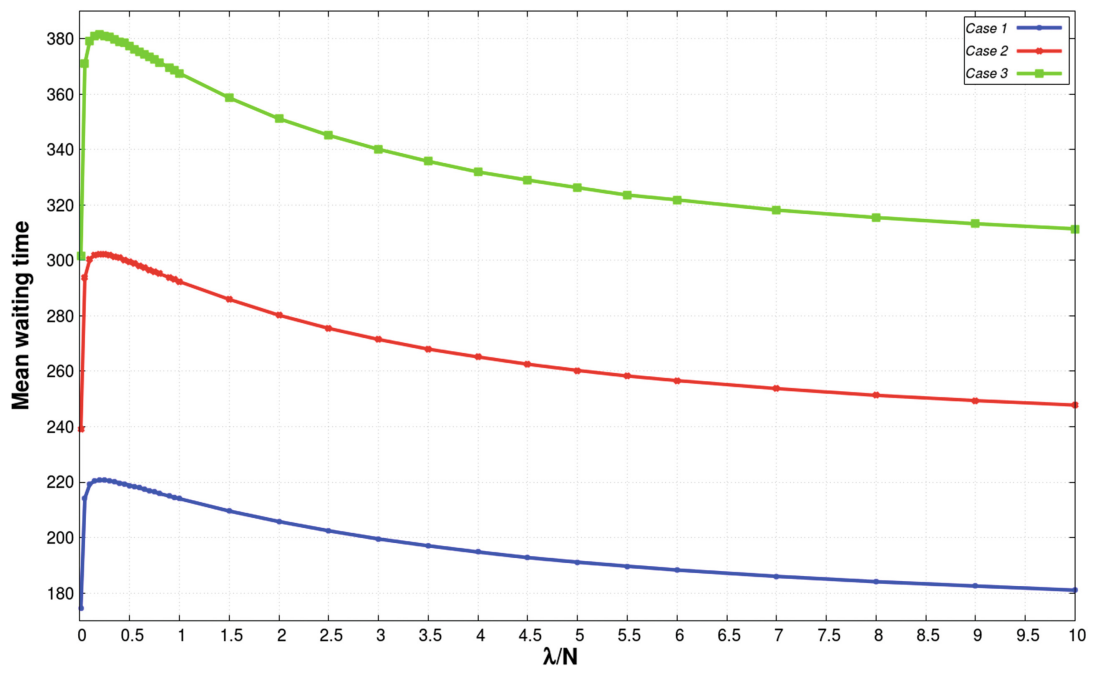

Fig. 3. Mean waiting time vs. intensity of incoming customers

Figure 3 represents the conformation of mean waiting time. The same parameters are (see Table 1) used as in case of Fig. 2 but here the running parameter is $\lambda / N$. As it is expected with the increment of $\lambda / N$ mean waiting time increases as well but an interesting phenomenon is noticeable namely after $\lambda / N$ is greater than 0.1 mean waiting time starts to decrease.

Figure 4 shows the effect of the inter-arrival time on the mean successful service time. Because of the opportunity of collisions it can easily happen that one 


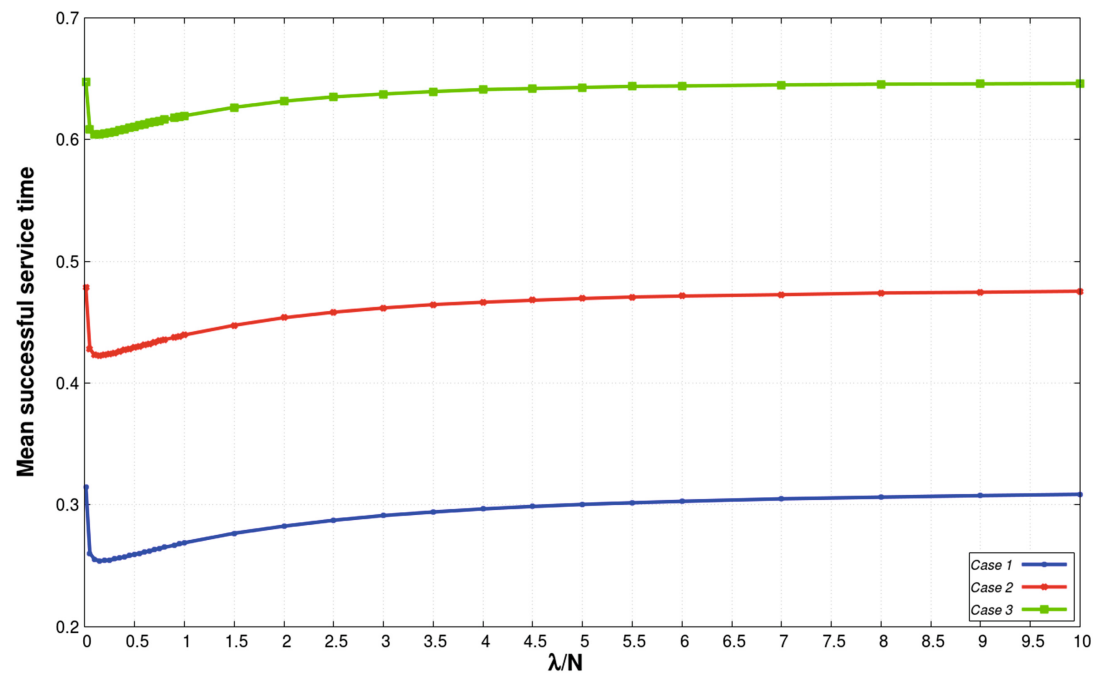

Fig. 4. Mean successful service time vs. intensity of incoming customers

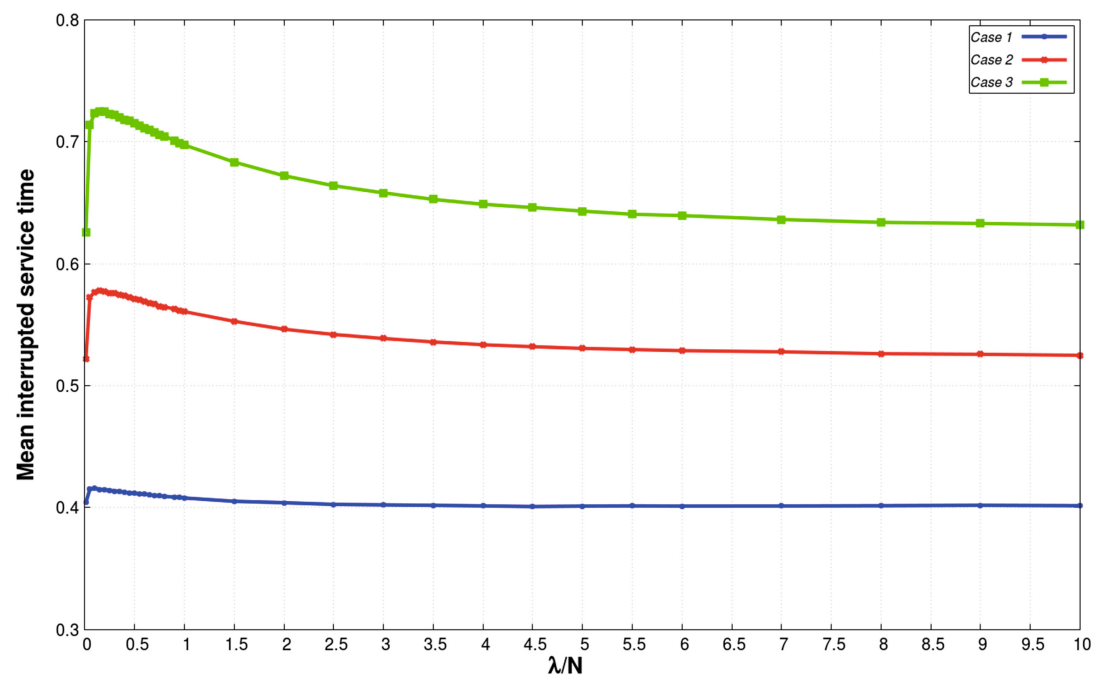

Fig. 5. Mean interrupted service time vs. intensity of incoming customers

job occurs multiple times in the service unit. Under mean successful service time we mean the average of those specific intervals when jobs are served appropriately. When $\lambda / N$ is 0.01 the mean successful time is the highest then it starts to decrease as $\lambda / N$ is increasing. This lasts till $\lambda / N$ reaches 0.1 , after it starts to increase.

On Fig. 5 the development of the mean interrupted service time can be seen. The opposite process happens compared to Fig. 4. It is worth mentioning when 
the service time is exponentially distributed then the sum of the mean interrupted service time and the mean successful service time is equal to $1 /$ rate parameter.

\subsection{Scenario B}

Scenario B is very similar to Scenario A except that now the distribution of inter-arrival times of the customers is not exponential but gamma distributed. The next table (see Table 3 ) presents the input parameters of Scenario B.

Table 3. Numerical values of parameters of Scenario B

\begin{tabular}{l|l|l|l|l|l|l|l|l|l}
\hline Case & $\mathrm{N}$ & $\alpha$ & $\beta$ & $\gamma_{0}$ & $\gamma_{1}$ & $\gamma_{2}$ & $\sigma / N$ & $\alpha_{1}$ & $\beta_{1} / N$ \\
\hline 1 & 100 & 1 & 1 & 0.1 & 0.1 & 1 & 0.01 & 0.5 & 0.01 \\
\hline 2 & 100 & 1 & 1 & 0.1 & 0.1 & 1 & 0.01 & 1 & 0.01 \\
\hline 3 & 100 & 1 & 1 & 0.1 & 0.1 & 1 & 0.01 & 2 & 0.01 \\
\hline
\end{tabular}

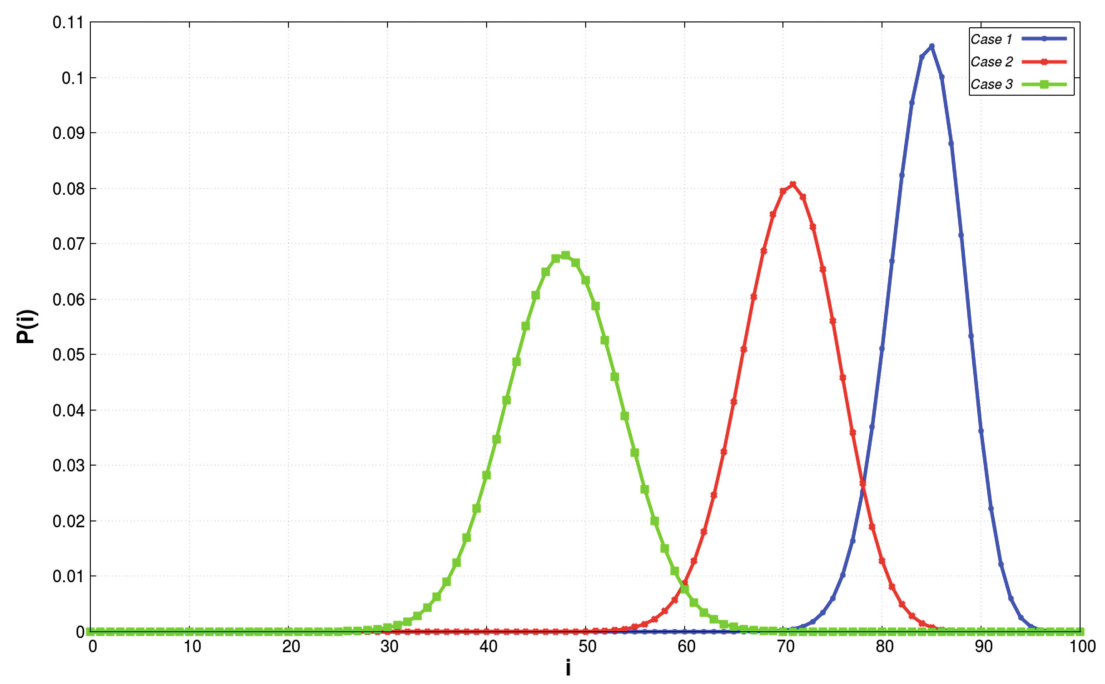

Fig. 6. Steady-state distributions of Scenario B

Figure 6 displays the steady-state distribution of Scenario B. Now the service time is exponentially distributed $(\alpha=1)$ and the inter-arrival time is gamma distributed. In these cases the steady-state distributions are still normally distributed and when $\alpha_{1}$ is greater than 1 the mean number of requests in the 
Table 4. Numerical results

\begin{tabular}{l|l|l|l|r|l|r|r|r|l}
\hline Case & $\mathrm{E}(\mathrm{NS})$ & $D^{2}(N S)$ & $\mathrm{E}(\mathrm{T})$ & $D^{2}(T)$ & $\mathrm{E}(\mathrm{W})$ & $D^{2}(W)$ & $\mathrm{E}(\mathrm{S})$ & $D^{2}(S)$ & $\mathrm{E}(\mathrm{IS})$ \\
\hline 1 & 84.3609 & 14.1827 & 270.0351 & 128831.5059 & 269.0354 & 128420.4087 & 0.4502 & 0.2039 & 0.5495 \\
\hline 2 & 70.5912 & 24.3012 & 239.9734 & 105273.4267 & 238.9734 & 104918.6389 & 0.4784 & 0.2289 & 0.5217 \\
\hline 3 & 47.7859 & 34.2376 & 183.0164 & 69830.9728 & 182.0164 & 69573.992 & 0.5462 & 0.2982 & 0.4538 \\
\hline
\end{tabular}

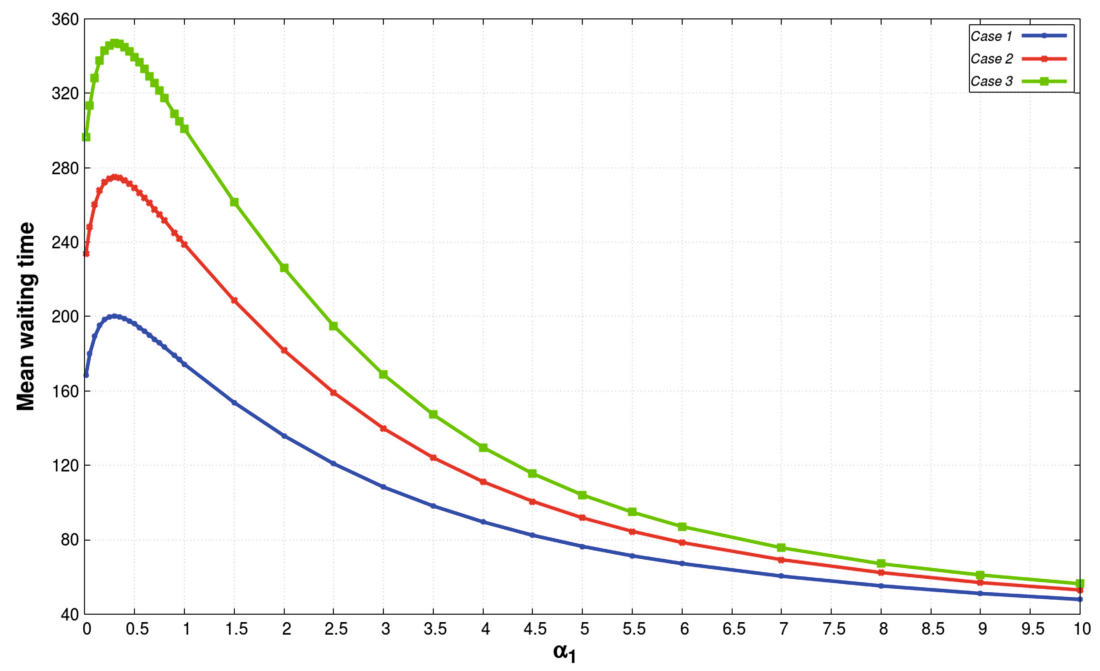

Fig. 7. Mean waiting time vs. shape parameter

system is significantly lower than in the other cases. In Table 7 the main performance measures can be found in connection with the cases (Table 4).

In Figs. 7, 8 and 9 the service time distribution of the Cases is the following (Table 5):

Table 5. Parameters of service time

\begin{tabular}{l|l|l}
\hline Case & $\alpha$ & $\beta$ \\
\hline 1 & 0.5 & 0.5 \\
\hline 2 & 1 & 1 \\
\hline 3 & 2 & 2
\end{tabular}

All the other parameters are according to Table 2. The running parameter is $\alpha_{1}$ so in this way the impact of different distributions on the various performance measures can be discovered. First the mean waiting time (Fig. 7), after an initial jump mean waiting time starts to monotonically decrease resulting that as $\alpha_{1}$ is getting bigger the less time the customers spend in the system. At the end the values of separate cases are almost equal. 


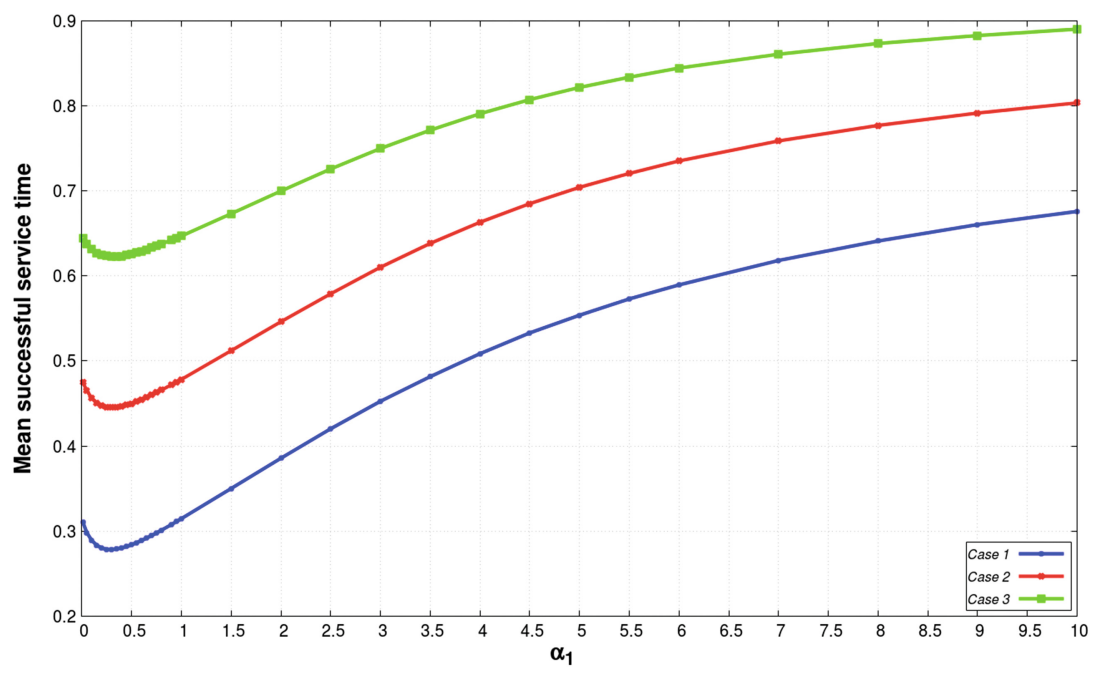

Fig. 8. Mean successful service time vs. shape parameter

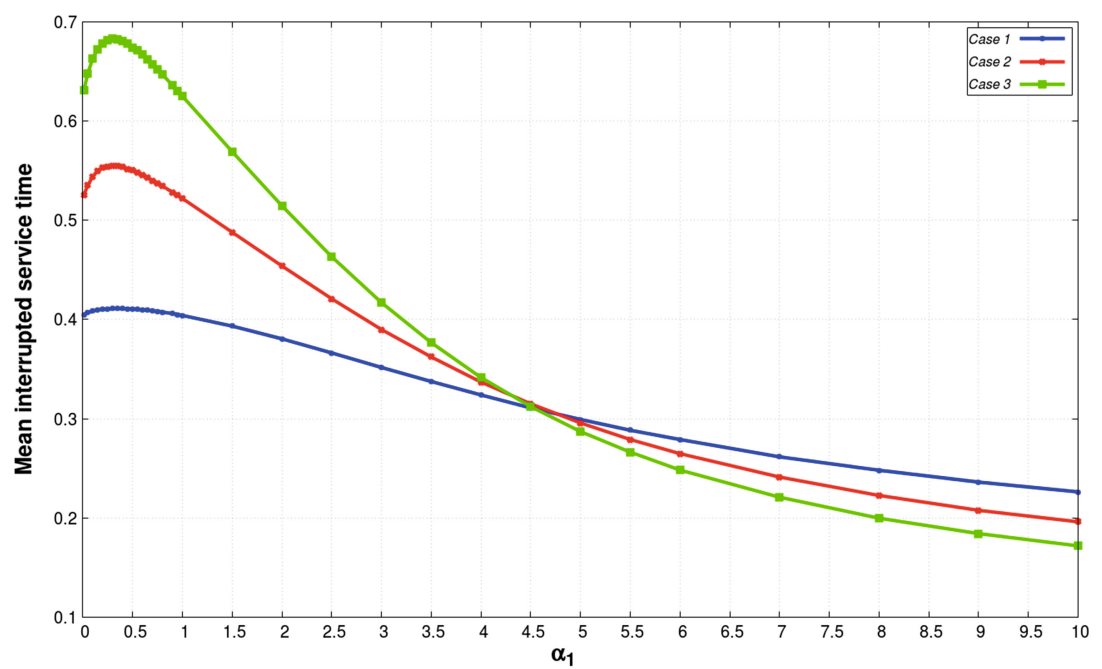

Fig. 9. Mean interrupted service time vs. shape parameter

As a consequence of Fig. 7 it is not surprising how the mean successful and interrupted service time change in function of $\alpha_{1}$. In those cases when the mean waiting time reaches the highest values the mean successful service time are the lowest. The mean interrupted service time acts the same as in case of the mean waiting time. It is interesting that after $\alpha_{1}$ is higher than 4.5 we observe that in Case 3 the values of the mean interrupted service time is the lowest among the 
Cases despite the fact that at the very start it possesses the highest values of mean interrupted service time.

\subsection{Scenario $\mathrm{C}$}

In Scenario $\mathrm{C}$ not just inter-arrival but also the retrial time is general distributed with the same parameters. Below Table 6 shows the input parameters of Scenario C.

Table 6. Numerical values of parameters of Scenario C

\begin{tabular}{l|l|l|l|l|l|l|l|l}
\hline Case & $\mathrm{N}$ & $\alpha$ & $\beta$ & $\gamma_{0}$ & $\gamma_{1}$ & $\gamma_{2}$ & $\alpha_{1}$ & $\beta_{1} / N$ \\
\hline 1 & 100 & 1 & 1 & 0.1 & 0.1 & 1 & 0.5 & 0.01 \\
\hline 2 & 100 & 1 & 1 & 0.1 & 0.1 & 1 & 1 & 0.01 \\
\hline 3 & 100 & 1 & 1 & 0.1 & 0.1 & 1 & 2 & 0.01 \\
\hline
\end{tabular}

Table 7 contains the main performance measures in connection with the cases.

Table 7. Numerical results

\begin{tabular}{l|l|l|l|r|r|r|r|r|l}
\hline Case & $\mathrm{E}(\mathrm{NS})$ & $D^{2}(N S)$ & $\mathrm{E}(\mathrm{T})$ & $D^{2}(T)$ & $\mathrm{E}(\mathrm{W})$ & $D^{2}(W)$ & $\mathrm{E}(\mathrm{S})$ & $D^{2}(S)$ & $\mathrm{E}(\mathrm{IS})$ \\
\hline 1 & 81.5384 & 16.9599 & 220.8314 & 82735.764 & 219.8314 & 82377.8317 & 0.3398 & 0.1183 & 0.6602 \\
\hline 2 & 70.5912 & 24.3012 & 239.9734 & 105273.4267 & 238.9734 & 104918.6389 & 0.4784 & 0.2289 & 0.5217 \\
\hline 3 & 56.6635 & 30.1636 & 261.483 & 146264.4778 & 260.4827 & 145919.0907 & 0.626 & 0.3915 & 0.3743 \\
\hline
\end{tabular}

This modification has no significant effect on the steady-state distribution (see Fig. 10). Of course the mean customers in the system is quite disparate but the distribution remains normal. Also when $\alpha_{1}$ is less than 1 it results higher mean number of customers in the system compared to when it is more than one.

As in earlier in Scenario B in Figs. 7, 8 and 9 the service time distribution of the Cases is the following (Table 8):

Table 8. Parameters of service time

\begin{tabular}{l|l|l}
\hline Case & $\alpha$ & $\beta$ \\
\hline 1 & 0.5 & 0.5 \\
\hline 2 & 1 & 1 \\
\hline 3 & 2 & 2
\end{tabular}




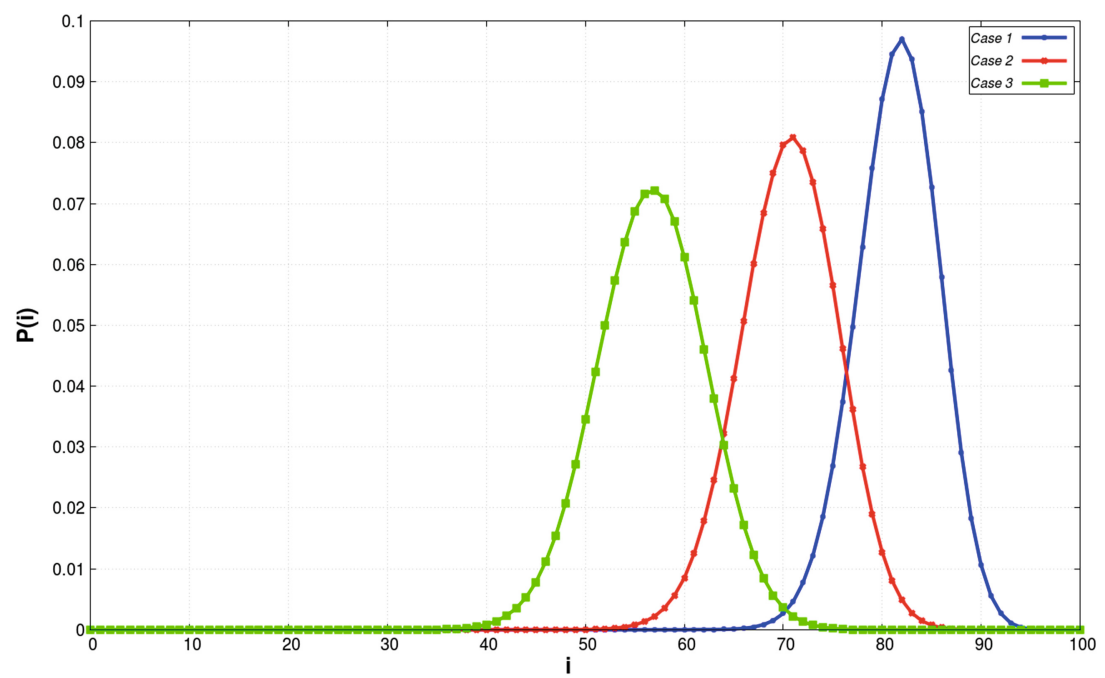

Fig. 10. Steady-state distributions of Scenario C

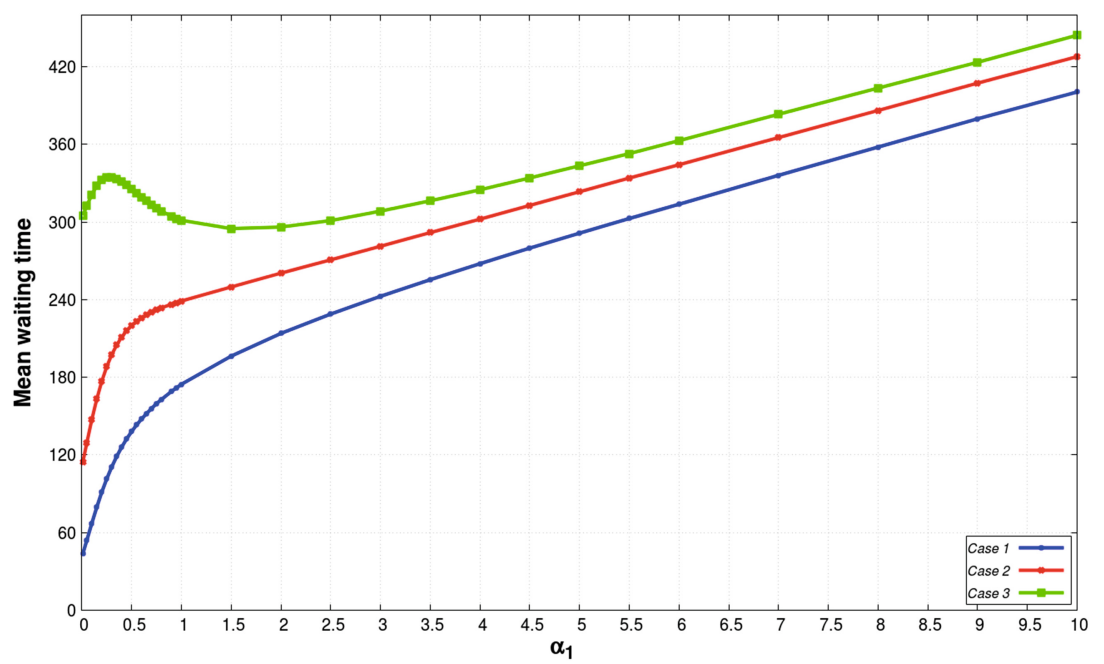

Fig. 11. Mean waiting time vs. shape parameter

Presently both inter-arrival and retrial distribution changes as $\alpha$ is increasing which results higher values of mean waiting time (Fig. 11). With the exception of Case 3 the others are monotonically increasing. This is true for the mean successful service time (Fig. 12) but now Case 3 rises from the beginning. 


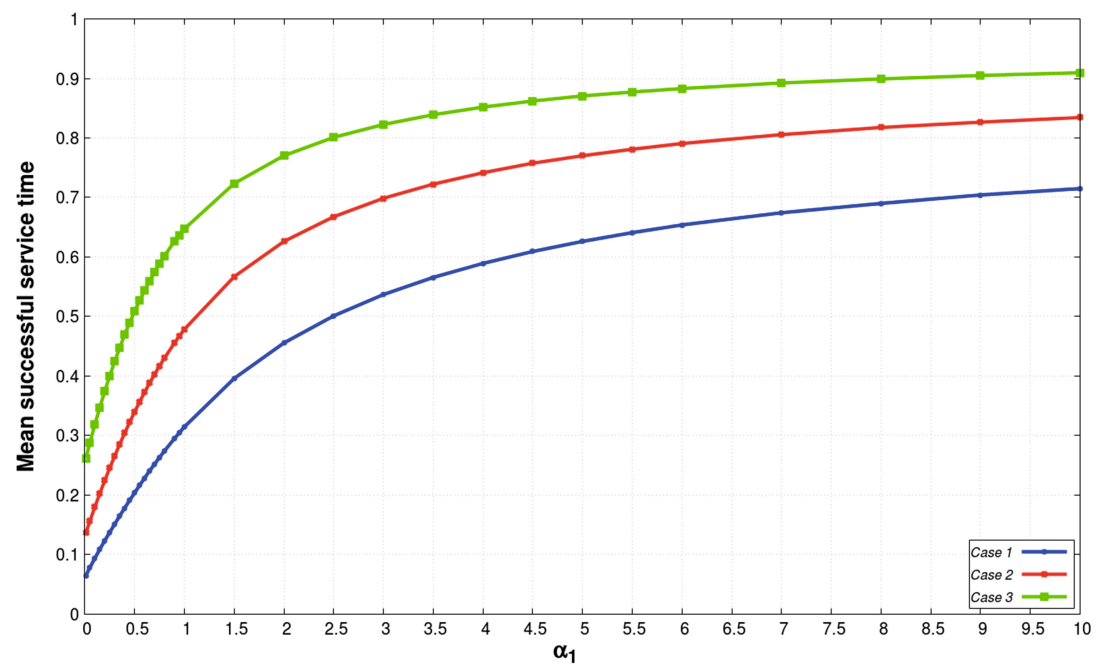

Fig. 12. Mean successful service time vs. shape parameter

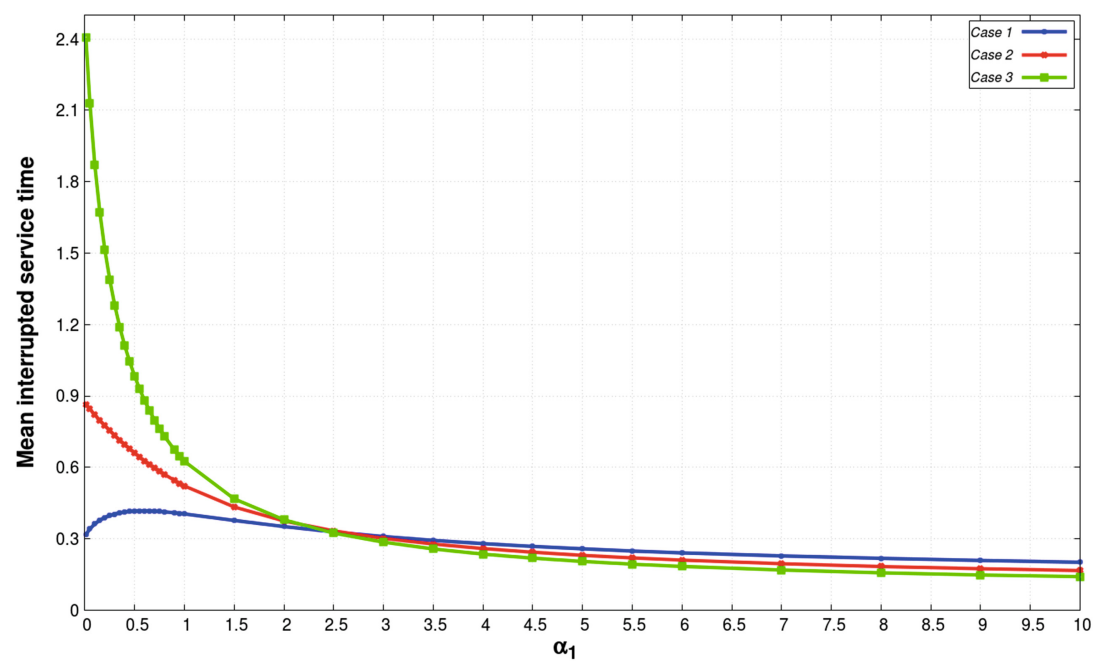

Fig. 13. Mean interrupted service time vs. shape parameter

The last figure shows the effect of the inter-arrival and inter-request time on the mean interrupted service time. At the beginning the difference is quite high among the Cases especially Case 3 has high values compared to the others but it is valid till $\alpha_{1}$ is smaller than 1 then the values of the Cases are nearly identical (Fig. 13). 


\section{Conclusions}

In this paper a finite-source retrial queueing model was introduced with unreliable server and the possibility of collision. In all cases in all scenarios it turns out that the steady-state distribution of the number of customers in the service facility is normally distributed. We used SimPack to carry out stochastic simulation showing the effect of different distribution of service, inter-arrival and retrial times on several main performance measures.

Acknowledgment. The work of Tamás Bérczes was supported in part by the project EFOP-3.6.2-16-2017-00015 supported by the European Union, co-financed by the European Social Fund.

\section{References}

1. Ali, A.A., Wei, S.: Modeling of coupled collision and congestion in finite source wireless access systems. In: 2015 IEEE Wireless Communications and Networking Conference (WCNC), pp. 1113-1118. IEEE (2015)

2. Almási, B., Roszik, J., Sztrik, J.: Homogeneous finite-source retrial queues with server subject to breakdowns and repairs. Math. Comput. Modelling 42(5-6), 673$682(2005)$

3. Artalejo, J.R., Gómez-Corral, A.: Retrial Queueing Systems. A Computational Approach. Springer, Berlin (2008)

4. Balsamo, S., Rossi, G.-L.D., Marin, A.: Modelling retrial-upon-conflict systems with product-form stochastic petri nets. In: Dudin, A., De Turck, K. (eds.) ASMTA 2013. LNCS, vol. 7984, pp. 52-66. Springer, Heidelberg (2013). doi:10. 1007/978-3-642-39408-9_5

5. Dragieva, V.I.: Number of retrials in a finite source retrial queue with unreliable server. Asia-Pac. J. Oper. Res. 31(2), 23 (2014)

6. Fishwick, P.A.: Simpack: getting started with simulation programming in C and C++. In: J.S. et al. (ed.) WSC 1992 Proceedings of the 24th Conference on Winter Simulation, pp. 154-162. ACM, New York (1992)

7. Gharbi, N., Dutheillet, C.: An algorithmic approach for analysis of finite-source retrial systems with unreliable servers. Comput. Math. Appl. 62(6), 2535-2546 (2011)

8. Kim, J., Kim, B.: A survey of retrial queueing systems. Ann. Oper. Res. 247(1), 3-36 (2016)

9. Kim, J.S.: Retrial queueing system with collision and impatience. Commun. Korean Math. Soc. 25(4), 647-653 (2010)

10. Kvach, A., Nazarov, A.: Sojourn time analysis of finite source markov retrial queuing system with collision. In: Dudin, A., Nazarov, A., Yakupov, R. (eds.) ITMM 2015. CCIS, vol. 564, pp. 64-72. Springer, Cham (2015). doi:10.1007/ 978-3-319-25861-4_6

11. Nazarov, A., Kvach, A., Yampolsky, V.: Asymptotic analysis of closed markov retrial queuing system with collision. In: Dudin, A., Nazarov, A., Yakupov, R., Gortsev, A. (eds.) ITMM 2014. CCIS, vol. 487, pp. 334-341. Springer, Cham (2014). doi:10.1007/978-3-319-13671-4_38 
12. Peng, Y., Liu, Z., Wu, J.: An M/G/1 retrial G-queue with preemptive resume priority and collisions subject to the server breakdowns and delayed repairs. J. Appl. Math. Comput. 44(1-2), 187-213 (2014). doi:10.1007/s12190-013-0688-7

13. Wang, J., Zhao, L., Zhang, F.: Analysis of the finite source retrial queues with server breakdowns and repairs. J. Ind. Manag. Optim. 7(3), 655-676 (2011)

14. Zhang, F., Wang, J.: Performance analysis of the retrial queues with finite number of sources and service interruptions. J. Korean Stat. Soc. 42(1), 117-131 (2013) 